\title{
The Effect of Psycho-Education on Depression, Anxiety and Pain for Patients Undergoing Hip And Knee Replacement
}

\author{
Hanan Faried Maghawry ${ }^{1}$, Alaa El Din Mohamed Darweesh ${ }^{2}$, Yaser Emam Mohamed ${ }^{3}$, Hosnia Shehata \\ Mohamed ${ }^{4}$. \\ 1- Demonstrator of Psychiatric\& Mental Health Nursing - Faculty of Nursing- Assiut University ${ }^{(1)}$, \\ 2- Professor of Psychiatry - Faculty of Medicine- Assiut University ${ }^{(2)}$, \\ 3- Professor of orthopedic surgery- Faculty of Medicine- Assiut University ${ }^{(3)}$, \\ 4- Lecturer of Psychiatric\& Mental Health Nursing - Faculty of Nursing- Assiut University ${ }^{(4)}$.
}

\begin{abstract}
Hip and knee replacement can be worrying, compromising the patient both physically and psychologically. Preoperative Psycho-education supports the success of interventions in reducing depression, anxiety and pain. The study aimed to: assess the effect of psycho-education on depression, anxiety and pain for patients undergoing hip and knee replacement. Subjects and Method: Pretest - posttest control group design was used. Purposive sample follows inclusion criteria included 90 patients; Preoperative psycho-education was applied for 43 patient as experimental group and another 47 patient didn't receive psycho-education as control group .Tools: patients were assessed through personal data sheet, Hospital Anxiety and Depression Scale (HADS) and Visual Analogue Scale (VAS). Results: both experimental and control group have high level of depression, anxiety and pain, there was significant improvement in levels of depression, anxiety and pain in the experimental group than control group after one month of operation. The study concluded that, preoperative psycho-education could be of high benefit in reducing depression, anxiety and pain for patients undergoing hip and knee replacement. Recommendation, Liaison psychiatric nurse, Psycho educational and relaxation training should be available to deal with the anxiety and depression.
\end{abstract}

Key words: Psycho-Education, Depression, Anxiety, Pain, Hip \& Knee Replacement.

\section{Introduction}

Knee replacement surgery (arthroplasty) is a routine operation that involves replacing a damaged, worn or diseased knee with a synthetic joint (Tang et al, 2016) Hip replacement is a surgical technique in which the hip joint is replaced by a prosthetic implant, that is, a hip prosthesis. Hip replacement surgery can be implemented as a total replacement or a hemi replacement (Hoefnagels, 2014).

Hip and knee replacement can be stressful emotionally and physically. It is thought that individuals, who receive information and are educated about their surgery and recovery before their surgery, will be less anxious and be able to handle pain better (Kisner et al., 2017).

Patients with the most manifest anxiety and depression reported the highest pre-operative scores for pain. This indicates that anxiety and depression may increase pain, making decisions about the indications for surgery difficult. This does not suggest that patients with anxiety and depression should be deprived of hip and knee replacement. May be the focus of initial treatment in these patients should be upon their mental state (Krupic et al., 2016). Pain associated with a range of chronic physical conditions is a significant contributing factor to increased rates of depression .About 20\% prevalence of depressive mood have been documented among osteoarthritis populations seeking specialist care (Hanusch et al., 2014).

Preoperative psycho-education is an educational intervention delivered before surgery that aims to improve people's knowledge, health behaviors and health outcomes (Boon et al., 2016) Pre-operative education includes the process of informing patients about their condition, surgery and post-operative care, and instruction about compliance to a treatment regime. Provided information about every aspect of hospitalization this included steps to minimize complications, teaching exercises that would increase post-operative function, and quality discharge planning (Nawana et al., 2015) Depression and anxiety levels can be reduced by pre-operative education, also pre-operative 'psycho-educational interventions' influence post-operative outcomes. And has positive effects in Patients' psychological well-being, including reduced anxiety and depression (Simeone et al., 2017) The role of the nurse in the care of the hip and knee replacement patient is to educate, provide safe and competent care, and help coordinate the care provided by the multidisciplinary team. The nurse is a critical link in providing the continuity of care required for these patients throughout the preoperative phase (Rothrock, 2014). This study aimed to assess the effect of psycho- 
education on depression, anxiety and pain for patients undergoing hip and knee replacement.

\section{Significance of the study}

There are more than 1 million hip and knee replacements in United States and about 160,000 in England and Wales per year (Hafez \& Sameh, 2016). And about 360 hip and knee replacements at Assiut university per year (Assiut University Hospital).

Anxiety and depression are interrelated with pain and physical limitations among patients undergoing Total Knee Replacement (TKR) and Total Hip Replacement (THR). Studies showed that psycho educational preparation for surgery supports the effectiveness of interventions in reducing pain and anxiety (Blom et al., 2016).

Preoperative psychological problems may affect physical recovery, well-being and postsurgical pain (Chen et al., 2014) So, the present study could be helpful in providing preoperative psycho education before THR and TKR to decrease patients' psychological problems, in order to inform patients about expected rates of progress, shorten the length of the hospital stay and decrease the health care costs.

\section{Aim of the study}

Assess the effect of psycho-education on depression, anxiety and pain for patients undergoing hip and knee replacement.

\section{Research Hypothesis}

Patients who receive psycho-education before surgery will have less post-operative depression, anxiety and pain than who don't receive.

\section{Material and methods}

\section{Research design}

Quantitative approach; quasi experimental, Pretest posttest control group design.

\section{Setting}

The study was carried out at Orthopedic Surgery Department at Assiut University Hospital which serves Assiut governorate and other surrounding area.

\section{Sample}

Non probability (purposive) sample was utilized, consisting of 100 patients; 50 patients undergone hip and knee replacement surgery as experimental group and from which 7 patients dropped out during follow up evaluation and became 43 patients as experimental group and 50 patients undergone hip and knee replacement surgery as control group and 3 patients dropped out during follow up evaluation and became 47 patients as control group. Drop out due to inability of the researcher to communicate with patients. The study sample became 90 patients; Preoperative psycho-education was applied for experimental group and other patients didn't receive psycho-education as control group. The sample size was conducted according to these inclusion criteria, Patients who accepted to participate in the study, with no history of chronic and debilitating illness, no psychoactive substance use either history or positive screening and no positive history of psychiatric disorder. The study was conducted in 9 months period from October 2017 to June 2018.

\section{Study Tools}

\section{Each participant was evaluated through the} following tools

\section{1- Socio demographic data.}

This tool was developed by the researcher to collect the information about the Socio Demographic data such as (name, age, gender, educational level, residence, marital status, occupation and type of operation).

2- Hospital Anxiety \& Depression Scale (HADS):This scale was originally developed by (Zigmond \& Snaith ,1983) and translated into Arabic version by(El-Rufaie \& Absood, 1987) HADS contains 14 items assessing anxiety (7-item) and depression (7-item), which are graded on a 4point Likert-type (from 0 to 3 ). The scores in each subscale are calculated by summing the conforming items, with maximum scores of 21 for each subscale. A score of $0-7$ is considered as normal, 8-10 as a borderline case and 11-21 as a case (anxiety or depression).

Reliability: Cronbach's alpha showed strong reliability with standardized alpha of 0.87 among 14 items.

Validity: the scale possesses high validity by using Pearson correlation. It is $93 \%$ for the total scale.

3- A Visual Analogue Scale (VAS):-This scale was originally developed by (Mottola,1993). A Visual Analogue Scale (VAS) is a measurement instrument that tries to measure a characteristic or attitude that is believed to range across a range of values and cannot easily be directly measured. Using a ruler, the score is determined by measuring the distance $(\mathrm{mm})$ on the $10-\mathrm{cm}$ line between "no pain" and "severe pain" and the patient's mark, providing a range of scores from 0-100. A higher score indicates greater pain intensity. The following cut points on the pain have been recommended: no pain $(0-4 \mathrm{~mm})$, mild pain $(5-44 \mathrm{~mm})$, moderate pain $(45-74 \mathrm{~mm})$, and severe pain $(75-100 \mathrm{~mm})$.

\section{Procedure}

1. Assessment phase

After approval of the study by The Dean of faculty of Nursing and the Head of orthopedic surgery department .patient interviewed individualized At the beginning to verify the study protocol and take informed consent, patient as well as the researcher were introduced to each other and the assessment 
sheet (pre -test) for depression, anxiety and pain was provided before starting psycho-education and before surgery for the experimental and control group.

\section{2-Implementation phase}

- for experimental group, The researcher implemented psycho-education session which included, an introduction, goals, causes, complications of hip replacement surgery, rehabilitation after hip and knee replacement surgery and providing relaxation technique (deep breathing, progressive relaxation technique and meditation to enhance relaxation) were implemented after modeling and under supervision of the researcher. Psycho-education included use of booklets, videos, and pictures.

- A pre-designed booklet by the researcher was given to each patient undergoing hip and knee replacement before surgery. The illustrated booklet had brief information about the nature of surgery and expectations after surgery. It included pre- and postoperative exercises and instructions.

- because some patients was admitted for one to two days maximum before surgery so that the researcher met the patients one session before surgery lasted from 45 minutes to one hour, providing psychoeducation for each patient individualized, the researcher discussed the content to them and answers their questions.

-As regards control group, didn't receive psychoeducation.

\section{3- Evaluation phase:}

- For experimental and control group, Communication continued with the patient after operation for follow up.

-Tools sheet (Hospital Anxiety and Depression scale HADS and Visual Analogue Scale VAS) were filled by subjects and recorded by the researcher. The patient reevaluate in outpatient clinic or by telephone to evaluate the effect of psychoeducation.

\section{Pilot Study}

A pilot study was carried out on a group of ten patients (included in the study sample) at orthopedic surgery department in Assiut University Hospital to test the clarity and objectivity of the questions.

\section{Ethical considerations:-}

- Research proposal approved from ethical committee in the faculty of nursing.

- There was no risk for the study subjects during application of the research.

- The study followed common ethical principles in clinical research.

- Patients have the right to refuse participating and/ or withdrawing from the study without any rationale at any time.
- Informed oral consent was obtained from every patient to participate in the study after explaining the purpose of the study.

- Privacy and confidentiality were assured during the whole study steps.

\section{Statistical Analysis}

The data were computerized and verified using the SPSS (Statistical Package for Social Science) version 20 to made tabulation and statistical analysis. For quantitative data, the frequencies, percentages, paired t-test, Pearson correlation coefficient, mean and standard deviation were calculated. For qualitative data, comparison between two groups and more was done using Chi-square test $\left(\chi^{2}\right)$. P- Value is considered significant if it was less than 0.05 . 


\section{Result}

Table (1): Socio-demographic characteristics of patients undergoing hip and knee replacement $(\mathrm{No}=90)$, (Experimental 43, control 47).

\begin{tabular}{|c|c|c|c|c|c|c|}
\hline \multirow[t]{2}{*}{ Variables } & \multicolumn{2}{|c|}{$\begin{array}{c}\text { Experimental group } \\
(\text { no.43) }\end{array}$} & \multicolumn{2}{|c|}{ Control group(no.47) } & \multirow{2}{*}{$\begin{array}{c}\text { t-test } \\
\chi^{2}\end{array}$} & \multirow[t]{2}{*}{ P -value } \\
\hline & No. & $\%$ & No. & $\%$ & & \\
\hline Age group & & & & & \multirow{4}{*}{4.890} & \\
\hline$<60$ & 25 & 58.1 & 30 & 63.8 & & \\
\hline$>60$ & 18 & 41.9 & 17 & 36.2 & & 0.299 \\
\hline $\begin{array}{l}\text { Age } \\
\text { Range } \\
\text { Mean } \pm \text { SD }\end{array}$ & \multicolumn{2}{|c|}{$\begin{array}{c}29-80 \\
54.44 \pm 12.00 \\
\end{array}$} & \multicolumn{2}{|c|}{$\begin{array}{c}17-86 \\
54.45 \pm 14.92\end{array}$} & & \\
\hline \multicolumn{7}{|l|}{ Gender } \\
\hline Male & 13 & 30.2 & 17 & 36.2 & 0.356 & 0.551 \\
\hline Female & 30 & 69.8 & 30 & 63.8 & & \\
\hline \multicolumn{7}{|l|}{ Marital status } \\
\hline Single & 4 & 9.3 & 4 & 8.5 & & \\
\hline Married & 35 & 81.4 & 33 & 70.2 & 2.810 & 0.422 \\
\hline Divorced & 0 & 0.0 & 1 & 2.1 & & \\
\hline Widow & 4 & 9.3 & 9 & 19.1 & & \\
\hline \multicolumn{7}{|l|}{ Residence } \\
\hline Rural & 33 & 76.7 & 33 & 70.2 & .490 & 0.484 \\
\hline Urban & 10 & 23.3 & 14 & 29.8 & & \\
\hline \multicolumn{7}{|l|}{ Occupation } \\
\hline House wife & 31 & 72.1 & 28 & 59.6 & 9.175 & 0.010 \\
\hline Worker & 12 & 27.9 & 10 & 21.3 & & \\
\hline Employee & 0 & 0.0 & 9 & 19.1 & & \\
\hline \multicolumn{7}{|l|}{ Educational level } \\
\hline Illiterate & 24 & 55.8 & 25 & 53.2 & 2.218 & 0.696 \\
\hline Read and write & 10 & 23.3 & 11 & 23.4 & & \\
\hline Secondary & 9 & 20.9 & 11 & 23.4 & & \\
\hline
\end{tabular}

Table (2): Frequency of pain intensity according to Visual Analogue Scale (VAS) preoperative and follow up for experimental group $(\mathrm{N}=43)$ and controls $(\mathrm{N}=47)$

\begin{tabular}{|c|c|c|c|c|c|c|c|c|c|c|}
\hline \multirow{3}{*}{ VAS } & \multicolumn{4}{|c|}{ Experimental group (no.43) } & \multirow{3}{*}{$\begin{array}{c}P \\
\text { Value }\end{array}$} & \multicolumn{4}{|c|}{ Control group (no.47) } & \multirow{3}{*}{$\begin{array}{c}P \\
\text { Value }\end{array}$} \\
\hline & \multicolumn{2}{|c|}{ Preoperative } & \multicolumn{2}{|c|}{ Follow Up } & & \multicolumn{2}{|c|}{ Preoperative } & \multicolumn{2}{|c|}{ Follow Up } & \\
\hline & No. & $\%$ & No. & $\%$ & & No. & $\%$ & No. & $\%$ & \\
\hline No pain & 0 & 0.0 & 20 & 46.5 & & 0 & 0.0 & 6 & 12.8 & \\
\hline Mild & 0 & 0.0 & 13 & 30.2 & 0.001 & 5 & 10.6 & 10 & 21.3 & 0.001 \\
\hline Moderate & 12 & 27.9 & 5 & 11.6 & & 14 & 29.8 & 18 & 38.3 & \\
\hline Severe & 31 & 72.1 & 5 & 11.6 & & 28 & 59.6 & 13 & 27.7 & \\
\hline
\end{tabular}

** Statistically significant difference ( $p$ 0.001) 
Table (3): Frequency of change in Hospital Anxiety and Depression scale (HADS) classification for experimental and control group preoperative and follow up evaluation.

\begin{tabular}{|c|c|c|c|c|c|c|c|c|c|c|}
\hline \multirow{3}{*}{ HADS } & \multicolumn{4}{|c|}{$\begin{array}{l}\text { Experimental } \\
\text { Group(no.43) }\end{array}$} & \multirow{3}{*}{ p-value } & \multicolumn{4}{|c|}{ Control group(no.47) } & \multirow{3}{*}{ p-value } \\
\hline & \multicolumn{2}{|c|}{ Preoperative } & \multicolumn{2}{|c|}{ Follow Up } & & \multicolumn{2}{|c|}{ Preoperative } & \multicolumn{2}{|c|}{ Follow Up } & \\
\hline & No. & $\%$ & No. & $\%$ & & No. & $\%$ & No. & $\%$ & \\
\hline \multicolumn{10}{|l|}{ Anxiety Scale } & \multirow{8}{*}{0.001} \\
\hline Normal & 0 & 0.0 & 41 & 95.3 & \multirow{7}{*}{0.001} & 5 & 10.6 & 11 & 23.4 & \\
\hline Borderline & 3 & 7.0 & 0 & 0.0 & & 9 & 19.1 & 11 & 23.4 & \\
\hline Case & 40 & 93.0 & 2 & 4.7 & & 33 & 70.2 & 25 & 53.2 & \\
\hline \multirow{4}{*}{$\begin{array}{l}\text { Depression } \\
\text { Scale } \\
\text { Normal } \\
\text { Borderline } \\
\text { Case }\end{array}$} & & & & & & & & & & \\
\hline & 0 & 0.0 & 35 & 81.4 & & 27 & 57.4 & 15 & 31.9 & \\
\hline & 2 & 4.7 & 3 & 7.0 & & 9 & 19.1 & 22 & 46.8 & \\
\hline & 41 & 95.3 & 5 & 11.6 & & 11 & 23.4 & 10 & 21.3 & \\
\hline
\end{tabular}

** Statistically significant difference (p 0.001)

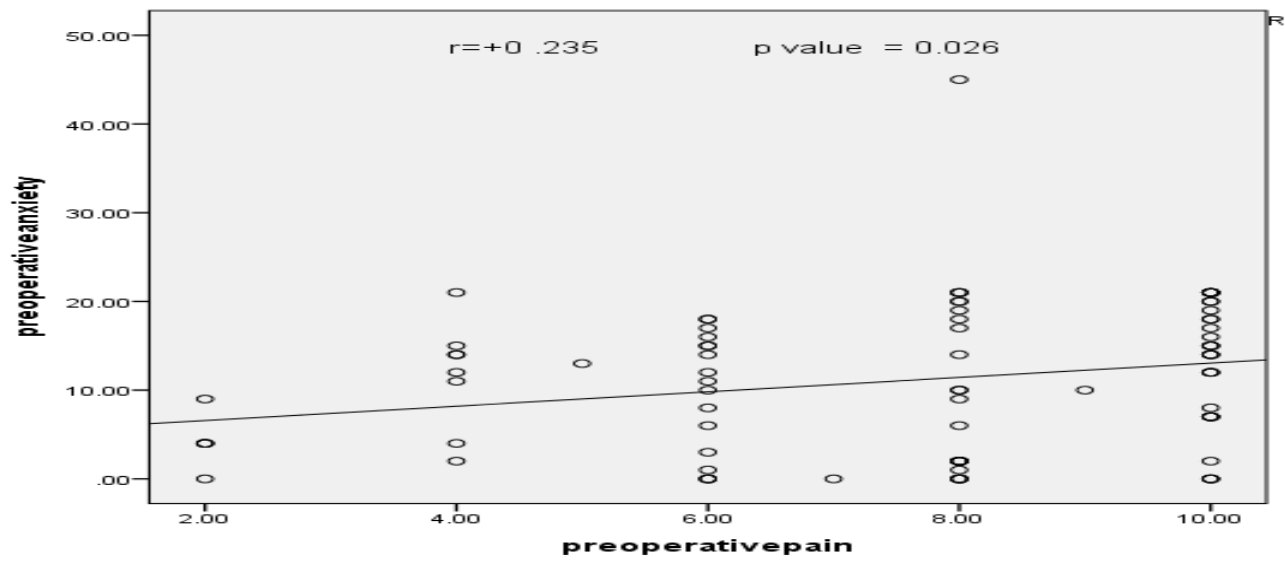

Figure (1): Correlation between preoperative Anxiety and preoperative pain:

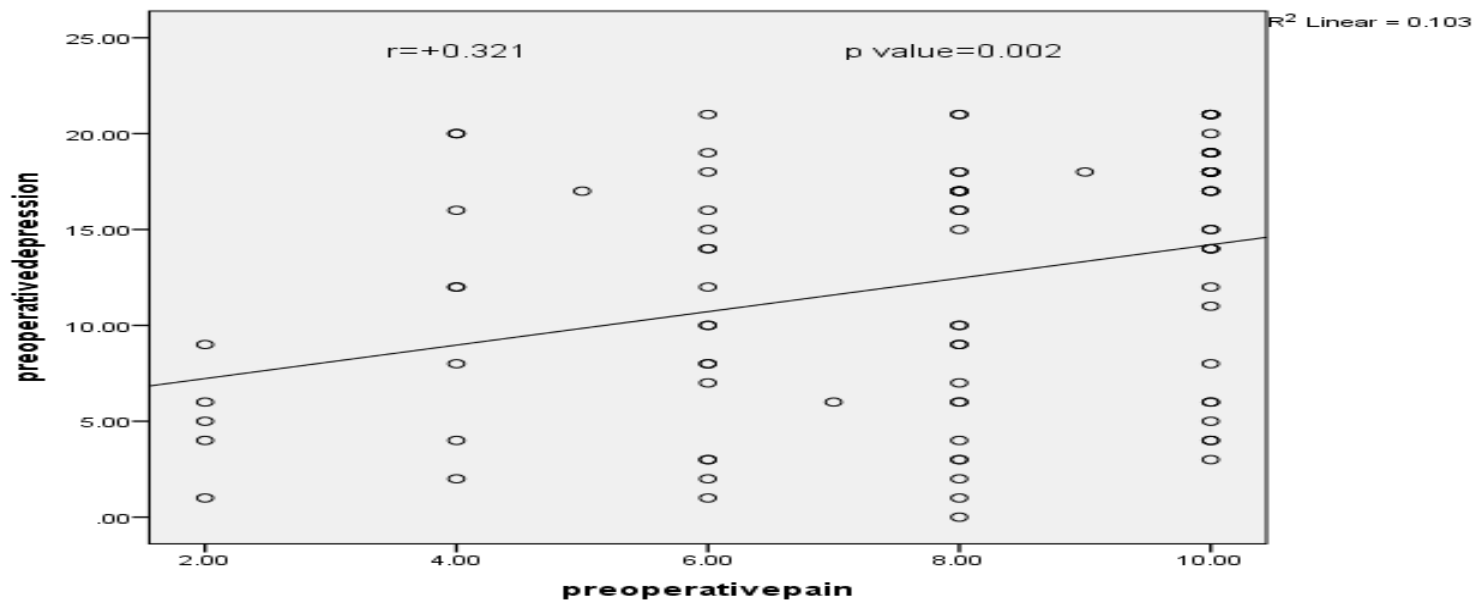

Figure (2): Correlation between preoperative depression and preoperative pain: 


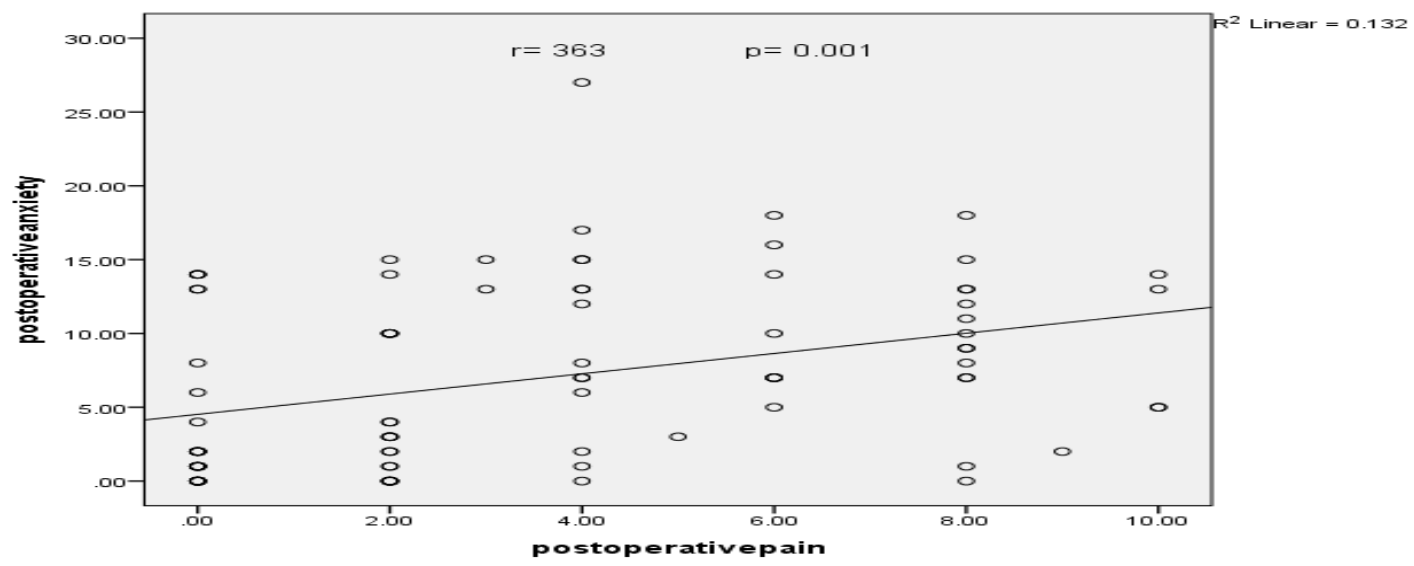

Figure (3): Correlation between postoperative Anxiety and postoperative pain:

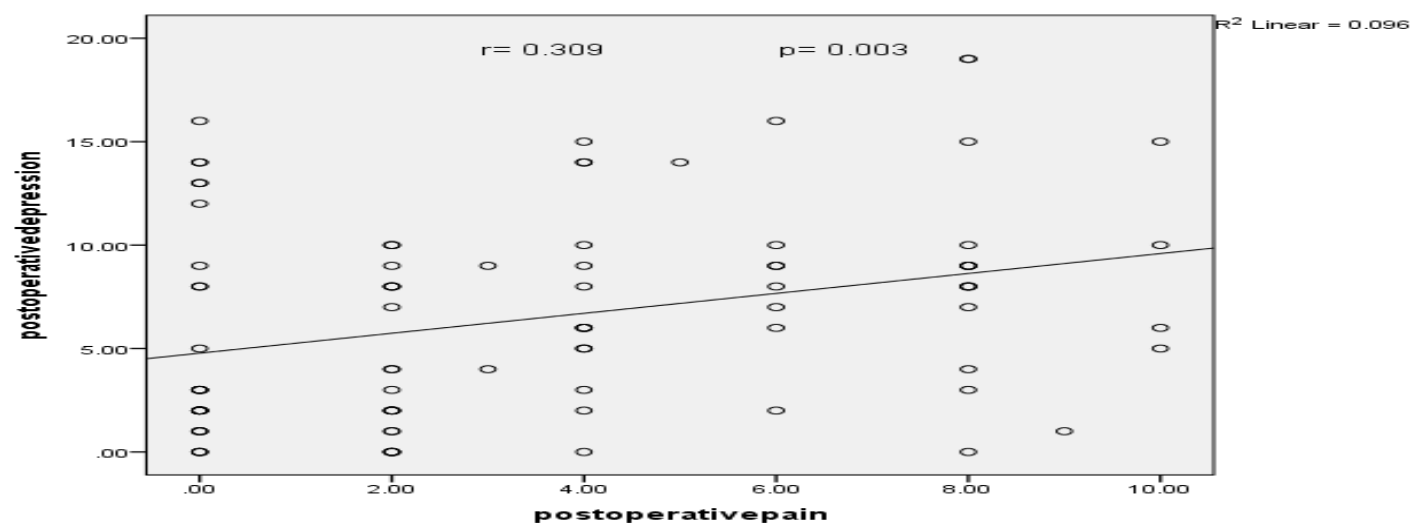

Figure (4): Correlation between postoperative depression and postoperative pain:

Table (4) Comparison between total mean score and improvement rate of Hospital Anxiety Scale for experimental and control group according to socio-demographic characteristics.

\begin{tabular}{|c|c|c|c|c|c|c|}
\hline \multirow{4}{*}{$\begin{array}{c}\text { Socio- } \\
\text { demographic } \\
\text { characteristics }\end{array}$} & \multicolumn{6}{|c|}{ Anxiety } \\
\hline & \multicolumn{3}{|c|}{ experimental group } & \multicolumn{3}{|c|}{ control group } \\
\hline & \multicolumn{2}{|c|}{ Mean \pm SD } & \multirow{2}{*}{$\begin{array}{c}\text { Improvement } \\
\text { Rate }\end{array}$} & \multicolumn{2}{|c|}{ Mean \pm SD } & \multirow{2}{*}{$\begin{array}{c}\text { Change } \\
\text { Rate }\end{array}$} \\
\hline & Preoperative & Follow up & & Preoperative & Follow up & \\
\hline Sex & & & & & & \\
\hline Male & $17.23 \pm 3.09$ & $1.92 \pm 1.80$ & $\mathbf{8 8 . 8 \%}$ & $3.00 \pm 5.06$ & $10.59 \pm 4.54$ & $71.6 \%$ \\
\hline Female & $17.67 \pm 6.46$ & $2.70 \pm 4.14$ & $83.7 \%$ & $6.53 \pm 4.99$ & $11.10 \pm 3.89$ & $41.8 \%$ \\
\hline P Value & \multicolumn{3}{|c|}{0.001} & \multicolumn{3}{|c|}{0.001} \\
\hline $\begin{array}{l}\text { Residence } \\
\text { Rural }\end{array}$ & $17.55 \pm 6.191$ & $2.55 \pm 4.83$ & $85.4 \%$ & $4.45 \pm 4.73$ & $10.88 \pm 4.51$ & $59.1 \%$ \\
\hline Urban & $17.50 \pm 3.37$ & $7.54 \pm 5.43$ & $56.9 \%$ & $7.14 \pm 6.09$ & $11.00 \pm 3.06$ & $36.4 \%$ \\
\hline P Value & \multicolumn{3}{|c|}{0.001} & \multicolumn{3}{|c|}{0.001} \\
\hline \multicolumn{7}{|l|}{ Occupation } \\
\hline House wife & $17.77 \pm 6.38$ & $2.97 \pm 5.36$ & $83.28 \%$ & $6.43 \pm 5.32$ & $10.61 \pm 4.29$ & $39.6 \%$ \\
\hline Worker & $16.92 \pm 3.00$ & $1.58 \pm 1.38$ & $90.66 \%$ & $3.60 \pm 5.85$ & $13.30 \pm 2.98$ & $72.9 \%$ \\
\hline Employee & - & - & - & $3.44 \pm 3.57$ & $9.22 \pm 3.70$ & $51.8 \%$ \\
\hline P Value & \multicolumn{3}{|c|}{0.001} & \multicolumn{3}{|c|}{0.001} \\
\hline
\end{tabular}




\begin{tabular}{|c|c|c|c|c|c|c|}
\hline \multirow{4}{*}{$\begin{array}{c}\text { Socio- } \\
\text { demographic } \\
\text { characteristics }\end{array}$} & \multicolumn{6}{|c|}{ Anxiety } \\
\hline & \multicolumn{3}{|c|}{ experimental group } & \multicolumn{3}{|c|}{ control group } \\
\hline & \multicolumn{2}{|c|}{ Mean \pm SD } & \multirow{2}{*}{$\begin{array}{c}\text { Improvement } \\
\text { Rate }\end{array}$} & \multicolumn{2}{|c|}{ Mean \pm SD } & \multirow{2}{*}{$\begin{array}{c}\text { Change } \\
\text { Rate }\end{array}$} \\
\hline & Preoperative & Follow up & & Preoperative & Follow up & \\
\hline $\begin{array}{l}\text { Marital status } \\
\text { Single }\end{array}$ & $16.50 \pm 4.20$ & $1.00 \pm 0.82$ & $93.9 \%$ & $7.00 \pm 6.48$ & $10.75 \pm 3.86$ & $34.6 \%$ \\
\hline Married & $17.03 \pm 3.62$ & $2.86 \pm 5.07$ & $83.1 \%$ & $5.03 \pm 5.05$ & $2.86 \pm 5.07$ & $42.8 \%$ \\
\hline Divorced & - & - & & $4.00 \pm 0.0$ & $10.00 \pm 0.0$ & $60 \%$ \\
\hline Widow & $23.00 \pm 15.23$ & $1.75 \pm 1.71$ & $92.3 \%$ & $5.44 \pm 6.25$ & $10.33 \pm 5.41$ & $47.3 \%$ \\
\hline P Value & \multicolumn{3}{|c|}{0.02} & \multicolumn{3}{|c|}{0.001} \\
\hline $\begin{array}{l}\text { Educational level } \\
\text { Illiterate } \\
\text { Read and Write } \\
\text { Secondary } \\
\end{array}$ & $\begin{array}{r}17.71 \pm 6.89 \\
17.40 \pm 2.72 \\
17.22 \pm 4.55 \\
\end{array}$ & $\begin{array}{l}2.96 \pm 5.54 \\
2.50 \pm 4.17 \\
1.67 \pm 1.87 \\
\end{array}$ & $\begin{array}{l}83.2 \% \\
85.6 \% \\
\mathbf{9 0 . 3 \%} \\
\end{array}$ & $\begin{array}{l}4.52 \pm 4.88 \\
6.73 \pm 5.73 \\
5.45 \pm 5.75 \\
\end{array}$ & $\begin{array}{l}10.68 \pm 4.43 \\
11.73 \pm 3.72 \\
10.64 \pm 3.91 \\
\end{array}$ & $\begin{array}{l}57.8 \% \\
\mathbf{4 6 . 8 \%} \\
49.2 \% \\
\end{array}$ \\
\hline P Value & \multicolumn{3}{|c|}{0.001} & \multicolumn{3}{|c|}{0.001} \\
\hline
\end{tabular}

Table (5) Comparison between total mean score and improvement rate of Hospital Depression Scale for experimental group and Change rate of depression for control group according to socio-demographic characteristics.

\begin{tabular}{|c|c|c|c|c|c|c|}
\hline \multirow{4}{*}{$\begin{array}{c}\text { Socio- } \\
\text { demographic } \\
\text { characteristics }\end{array}$} & \multicolumn{6}{|c|}{ Depression } \\
\hline & \multicolumn{3}{|c|}{ experimental group } & \multicolumn{3}{|c|}{ control group } \\
\hline & \multicolumn{2}{|c|}{ Mean \pm SD } & \multirow{2}{*}{$\begin{array}{c}\text { Improvement } \\
\text { Rate }\end{array}$} & \multicolumn{2}{|c|}{ Mean \pm SD } & \multirow{2}{*}{$\begin{array}{c}\text { Change } \\
\text { Rate }\end{array}$} \\
\hline & Preoperative & Follow up & & Preoperative & Follow up & \\
\hline Sex & & & & & & \\
\hline Male & $18.00 \pm 2.08$ & $3.08 \pm 3.75$ & $98.3 \%$ & $5.65 \pm 4.42$ & $8.71 \pm 3.50$ & $35.1 \%$ \\
\hline Female & $17.10 \pm 3.22$ & $4.13 \pm 4.85$ & $75.8 \%$ & $8.27 \pm 4.90$ & $9.00 \pm 4.35$ & $8.1 \%$ \\
\hline P Value & \multicolumn{3}{|c|}{0.001} & \multicolumn{3}{|c|}{0.001} \\
\hline Residence & & & & & & \\
\hline Rural & $17.36 \pm 3.01$ & $3.39 \pm 4.21$ & $80.4 \%$ & $7.15 \pm 5.05$ & $8.67 \pm 3.92$ & $17.5 \%$ \\
\hline Urban & $17.40 \pm 2.80$ & $5.20 \pm 5.45$ & $70.1 \%$ & $7.71 \pm 4.50$ & $9.43 \pm 4.36$ & $18.2 \%$ \\
\hline P Value & \multicolumn{3}{|c|}{0.001} & \multicolumn{3}{|c|}{0.001} \\
\hline \multicolumn{7}{|l|}{ Occupation } \\
\hline House wife & $17.23 \pm 3.24$ & $4.32 \pm 4.88$ & $40.2 \%$ & $8.43 \pm 4.80$ & $8.86 \pm 4.44$ & $4.8 \%$ \\
\hline Worker & $17.75 \pm 1.96$ & $2.50 \pm 3.26$ & $85.9 \%$ & $5.60 \pm 5.58$ & $10.30 \pm 4.03$ & $45.6 \%$ \\
\hline Employee & - & - & - & $5.78 \pm 3.46$ & $7.44 \pm 1.88$ & $22.3 \%$ \\
\hline P Value & \multicolumn{3}{|c|}{0.001} & \multicolumn{3}{|c|}{0.001} \\
\hline $\begin{array}{l}\text { Marital status } \\
\text { Single }\end{array}$ & $17.75 \pm 2.63$ & $1.75 \pm 1.26$ & $90.1 \%$ & $6.25 \pm 3.86$ & $5.75 \pm 2.06$ & $8 \%$ \\
\hline Married & $17.40 \pm 2.98$ & $4.00 \pm 4.86$ & $77 \%$ & $7.27 \pm 5.00$ & $9.48 \pm 4.10$ & $23.3 \%$ \\
\hline Divorced & - & - & - & 4.00 & $9.00 \pm 0.0$ & $55.5 \%$ \\
\hline Widow & $16.75 \pm 3.40$ & $4.25 \pm 3.40$ & $74.6 \%$ & $8.33 \pm 5.15$ & $8.11 \pm 4.14$ & $2.6 \%$ \\
\hline P Value & \multicolumn{3}{|c|}{0.02} & \multicolumn{3}{|c|}{0.001} \\
\hline $\begin{array}{l}\text { Educational level } \\
\text { Illiterate }\end{array}$ & $1683+290$ & $4.29+5.24$ & $745 \%$ & $7.16+4.90$ & $856+344$ & $163 \%$ \\
\hline Read and Write & $18.10 \pm 2.02$ & $2.70 \pm 2.7$ & $85 \%$ & $8.55 \pm 5.96$ & $10.91 \pm 4.72$ & $21.6 \%$ \\
\hline Secondary & $18.00 \pm 3.77$ & $3.78 \pm 4.27$ & $79 \%$ & $6.45 \pm 3.56$ & $7.64 \pm 4.15$ & $15.5 \%$ \\
\hline P Value & \multicolumn{3}{|c|}{0.001} & \multicolumn{3}{|c|}{0.001} \\
\hline
\end{tabular}


Table (6) Comparison between total mean score and improvement rate of Visual Analogue Scale according to socio-demographic characteristics for experimental and control group.

\begin{tabular}{|c|c|c|c|c|c|c|}
\hline \multirow{4}{*}{$\begin{array}{c}\text { Socio- } \\
\text { demographic } \\
\text { characteristics }\end{array}$} & \multicolumn{6}{|c|}{ VAS } \\
\hline & \multicolumn{3}{|c|}{ experimental group } & \multicolumn{3}{|c|}{ control group } \\
\hline & \multicolumn{2}{|c|}{ Mean \pm SD } & \multirow{2}{*}{$\begin{array}{l}\text { Improvement } \\
\text { Rate } \\
\end{array}$} & \multicolumn{2}{|c|}{ Mean \pm SD } & \multirow{2}{*}{$\begin{array}{c}\text { Improvemen } \\
\text { Rate }\end{array}$} \\
\hline & Preoperative & Follow up & & Preoperative & Follow up & \\
\hline Sex & & & & & & \\
\hline Male & $8.38 \pm 1.98$ & $1.23 \pm 1.30$ & $85.3 \%$ & $7.35 \pm 2.42$ & $4.41 \pm 3.37$ & $40 \%$ \\
\hline Female & $7.93 \pm 2.20$ & $2.50 \pm 3.29$ & $68.4 \%$ & $7.10 \pm 2.63$ & $5.00 \pm 2.68$ & $29.5 \%$ \\
\hline P Value & \multicolumn{3}{|c|}{0.001} & \multicolumn{3}{|c|}{0.001} \\
\hline Residence & & & & $721+236$ & $442+308$ & $386 \%$ \\
\hline \begin{tabular}{|l} 
Rural \\
Urhan
\end{tabular} & $\frac{8.36 \pm 1.97}{7.10+2.42}$ & $\frac{2.27 \pm 2.84}{160+3.10}$ & $\frac{72.8 \%}{77.4 \%}$ & $\frac{7.21 \pm 2.36}{7.14+3.01}$ & $\frac{4.42 \pm 3.08}{5.64+2.41}$ & $\frac{38.6 \%}{21 \%}$ \\
\hline \begin{tabular}{|l|} 
Urban \\
P Value
\end{tabular} & \multirow{2}{*}{\multicolumn{3}{|c|}{0.001}} & \multirow{2}{*}{\multicolumn{3}{|c|}{0.001}} \\
\hline P Value & & & & & & \\
\hline \multicolumn{7}{|l|}{ Occupation } \\
\hline Housewife & $8.00 \pm 2.19$ & $2.55 \pm 3.24$ & $68.1 \%$ & $7.43 \pm 2.50$ & $4.86 \pm 2.92$ & $34.4 \%$ \\
\hline Worker & $8.25 \pm 2.01$ & $1.00 \pm 1.04$ & $\mathbf{8 7 . 8 \%}$ & $7.40 \pm 1.35$ & $3.80 \pm 3.19$ & $48.6 \%$ \\
\hline Employee & - & - & - & $6.22 \pm 3.53$ & $5.67 \pm 2.65$ & $8.8 \%$ \\
\hline P Value & \multicolumn{3}{|c|}{0.001} & \multicolumn{3}{|c|}{0.002} \\
\hline $\begin{array}{l}\text { Educational } \\
\text { level }\end{array}$ & & & & & & \\
\hline Illiterate & $7.83 \pm 1.86$ & $2.17 \pm 2.88$ & $72.2 \%$ & $8.04 \pm 2.05$ & $4.84 \pm 2.87$ & $39.8 \%$ \\
\hline Read and Write & $8.20 \pm 2.57$ & $2.00 \pm 2.98$ & $75.6 \%$ & $7.18 \pm 2.32$ & $4.73 \pm 3.00$ & $34.1 \%$ \\
\hline Secondary & $8.56 \pm 2.40$ & $2.11 \pm 3.10$ & $75.3 \%$ & $5.27 \pm 2.87$ & $4.73 \pm 3.26$ & $10.2 \%$ \\
\hline P Value & \multicolumn{3}{|c|}{0.001} & \multicolumn{3}{|c|}{0.001} \\
\hline $\begin{array}{l}\text { Marital status } \\
\text { Single }\end{array}$ & $8.00 \pm 2.83$ & $3.00 \pm 3.83$ & $62.5 \%$ & $4.00 \pm 2.31$ & $5.00 \pm 4.16$ & - \\
\hline Married & $8.31 \pm 1.95$ & $1.86 \pm 2.79$ & $77.6 \%$ & $7.70 \pm 2.20$ & $5.18 \pm 2.93$ & $32.7 \%$ \\
\hline Divorced & - & - & - & $4.00 \pm 0.0$ & $2.00 \pm 0.0$ & $50 \%$ \\
\hline Widow & $6.00 \pm 2.31$ & $3.50 \pm 3.00$ & $41.6 \%$ & $7.11 \pm 2.85$ & $3.56 \pm 2.24$ & $49.9 \%$ \\
\hline P Value & \multicolumn{3}{|c|}{0.001} & \multicolumn{3}{|c|}{0.001} \\
\hline
\end{tabular}

Table (1): Illustrated that, the Experimental group included 43 patients, with mean age 54.44 years, $69.8 \%$ of them were females, $76.7 \%$ lived in rural area and $55.8 \%$ of them were illiterate. There were no significant differences among experimental and control groups as regard demographic characteristics except that high percentage of experimental group were house wife (72.1), $(\mathrm{p}=0.010)$.

Table (2): Showed that there were significant increase in percentages of patients with no or mild pain at follow up evaluation in experimental $(\mathrm{p}=0.001)$ group and in controls $(\mathrm{p}=0.001)$ group. However, the increase in no or mild pain in the experimental group are higher than their increase in control groups.

Table (3): Revealed that there were significant increase in percentages of patients with normal score of anxiety and depression at follow up evaluation in experimental $(\mathrm{p}=0.001)$ and in control $(\mathrm{p}=0.001)$ groups. However, the increase in frequency of normal score of anxiety and depression in the experimental group are higher than their increase in control groups.
Figure (1): Illustrated that there was significant positive correlation between preoperative anxiety and preoperative pain $\left(\mathrm{r}=0.235^{*}, \mathrm{p}=0.026\right)$.

Figure (2): Indicated that there was significant positive correlation between preoperative depression and preoperative pain $\left(\mathrm{r}=0.321^{*}, \mathrm{p}=0.002\right)$.

Figure (3): Illustrated that there was significant positive correlation between postoperative anxiety and postoperative pain $\left(\mathrm{r}=0.363^{*}, \mathrm{p}=0.001\right)$.

Figure (4): Indicated that there was significant positive correlation between postoperative depression and postoperative pain $\left(\mathrm{r}=0.309^{*}, \mathrm{p}=0.003\right)$.

Table (4): Showed that in the experimental group, improvement rate of anxiety was significantly high in male patients $(88.8 \%, \mathrm{p}=0.001)$, rural residents $(85.4$ $\%, \mathrm{p}=0.001)$, who are worker $(90.66 \%, \mathrm{p}=0.001)$, single $(93.9 \%, \mathrm{p}=0.001)$, and with secondary school education $(90.3 \%, \mathrm{p}=0.001)$. On the other hand control group showed increase of mean score of anxiety but still in borderline case except married patients showed improvement rate $(42.8 \%, \mathrm{p}=0.001)$. 
Table (5): Revealed that in the experimental group, improvement rate of depression is significantly high in male patients $(98.3 \%, \mathrm{p}=0.001)$, rural residents $(80.4 \%, \mathrm{p}=0.001)$, who are worker $(85.9 \%, \mathrm{p}=0.001)$, single $(90.1 \%, \mathrm{p}=0.02)$, and with read and write patients $(85 \%, \mathrm{p}=0.001)$. On the other hand control group showed increase of mean score of depression but still in borderline case except single patients showed improvement rate $(8 \%, \mathrm{p}=0.001)$.

Table (6): Demonstrated that in the experimental group, improvement rate of pain is significantly high in male patients $(85.3 \%, \mathrm{p}=0.001)$, urban residents (77.4\%, $\mathrm{p}=0.001)$, who are worker $(87.8 \%, \mathrm{p}=0.001)$, married $(77.6 \%, \mathrm{p}=0.001)$, and with read and write patients $(75.6 \%, \mathrm{p}=0.001)$. In the control group, improvement rate of pain is significantly high in male patients $(40 \%, \mathrm{p}=0.001)$, rural residents $(38.6 \%$, $\mathrm{p}=0.001)$, who are worker $(48.6 \%, \mathrm{p}=0.001)$, divorced $(50 \%, \mathrm{p}=0.001)$, and illiterate patients $(39.8 \%, \mathrm{p}=0.001)$.

\section{Discussion}

In The current study the patients who had replacement surgery and received preoperative psycho-education (the experimental group) had decreased post-operative depression, anxiety and pain than other patients (the control group) who didn't receive preoperative psycho-education.

This result is supported by Waimann et al., (2016) who reported that, an intervention could reduce the post-operative anxiety level from severe to mild. Also this result is consistent with finding of, Tristaino et al., (2016) who demonstrated that 12.8 $\%$ of patients in the experimental group revealed a state of anxiety, compared to $78.9 \%$ in the control group.

It could be explained that patients who receive preoperative psycho-education have information about the surgery, how to deal with the joint after surgery, and they were thoroughly informed about what they should do or don't to maintain the joint. They learned how to deal with pre and post-operative anxiety through learning types of relaxation techniques and they had a booklet as reference that contains all information about the surgery. However, other group of patients (control) who didn't receive preoperative psycho-education didn't know such information and were not prepared to deal with anxiety.

This study showed that, patients who had joint replacement surgery and received preoperative psycho-education had decreased depression than other patients who didn't receive preoperative psycho-education.

These results are supported by, Choi \& Ra, (2016) who reported decrease in depression after surgery. Similarly, Tristaino et al., (2016) found that, a state of postoperative depression was observed in $12.8 \%$ of patients in the experimental group and in $73.7 \%$ in the control group.

This might be related to that these patients had pathological preoperative anxiety and depression scores because whereas preoperative scores were high, whereas the prognosis was better. This is explained by the fact that informed and educated group knew that will experience such pain and knew how to deal with it and that led to a decrease in postoperative depression. They also knew signs and symptoms indicating possible complications about the surgery, which made them assured about the surgery rather than control group who didn't receive preoperative psycho-education.

The present study revealed that patients who had replacement surgery and received preoperative psycho-education had decreased pain other those who didn't receive preoperative psycho- education.

This is consistent with Lindberg et al., (2016) who found that, all of the post-operative pain scores were lower than the preoperative pain and interpreted this result by the intensive preoperative management that these patients received.

This may be related to that these patients had pathological preoperative pain scores because whereas preoperative scores were high, whereas the prognosis was better. And these patients who received pre-operative psycho-education had decrease in post-operative anxiety and depression that made them expect pain and knew how to deal with it and this preoperative psycho-education was effective in decreasing post-operative pain in experimental group who received preoperative psycho- education than control group who didn't receive preoperative psych-education.

The present study revealed that there are significant positive correlation between pre-operative anxiety and preoperative pain $\left(\mathrm{r}=0.235^{*}, \mathrm{p}=0.026\right)$. This is consistent with the study conducted by Burton et al., (2018) who found that there is relationship between preoperative anxiety and pain. Stoicea et al., (2018) also found that the presence of preoperative anxiety was associated with greater postoperative pain.

This explains the importance of reducing patients' anxiety as there is a positive relationship between anxiety and pain. Educational interventions can reduce anxiety and depression and increase patients' knowledge about the surgical process (Wilson et al., 2016).

This study found that there is significant positive correlation between pre-operative depression and preoperative pain $(\mathrm{r}=0.321 * \mathrm{p}=0.002)$.

With regards to preoperative depression, Stoicea et al., (2018) found that, presence of preoperative depression was associated with greater postoperative 
pain. However, Riddle et al., (2015) found that preoperative depressive symptoms were not prognostic of the extent of postoperative pain and that the extent of depressive symptoms was essentially unchanged after surgery. Choi \& Ra, (2016) indicated that patients who are depressed preoperatively have worse results of pain after knee replacement surgery.

This could be explained that patients with the most pronounced anxiety and depression reported the highest pre-operative scores for pain.

This suggests that anxiety and depression may increase pain, making decisions about the indications for surgery difficult. This does not suggest that patients with anxiety and depression should be denied surgery. Perhaps the focus of initial treatment in these patients should be upon their mental state (Krupic, et al., 2016).

Our results indicated that, there is highly significant association between gender and both anxiety and depression with highly improvement rate to females compared to males after surgery in experimental group. However there were no improvements in control group.

In contrast, Pasyar et al., (2018) found that the patient's sex was a risk factor for developing depression, with females more likely to become depressed post-operatively than males and although it did not influence postsurgical anxiety.

This can be explained that psycho-education was effective in improving anxiety and depression in experimental patients associated to sex compared to control group.

The current study reflected that, there is highly significant association between sex and pain with highly improvement rate to males after surgery in experimental compared to control group.

This result is congruent with the study conducted by, Hamilton et al., (2015) who indicated that female sex was associated with more postoperative pain than males.

This may be related to the effective psycho-education received by the experimental group that led to higher improvement to pain associated to gender from control group, and the hypersensitivity of females to pain compared to males.

This study revealed that there is highly significant association between level of education and anxiety with highly improvement rate to patients with secondary school education and with highly improvement rate of depression for patients who read and write in experimental group while there was no improvement of control group according to level of education. This finding is incongruent with the study conducted by Dailiana et al., (2015) who reported that low education was not a predictor of postoperative depression and anxiety.

This can be explained that the effectiveness of psycho-education the experimental group was received that led to highly improvement to patients who read and write and secondary school education. This can be interpreted by the fact that these patients are more able to search, read and ask about knowledge of disease process and interventions of surgery and use the booklet effectively than illiterate patients.

This study revealed that there is highly significant association between level of education and pain with higher improvement rate in read and write in experimental group and to illiterate patients in control group. This is congruent with the study conducted by, Dailiana et al., (2015) who reported that low education was not a predictor of post-operative pain. In contrast, Vina et al., (2017) indicated that patients with severe pain were characterized by lower level of education. On the other hand, Scott et al., (2016) reported that there isn't association between level of education and pain.

This can be explained that psycho-education was effective and led to higher improvement of pain in the experimental group than in control group.

In this study, there is highly significant association between residence and both anxiety and depression with highly improvement rate to patients live in rural area in the experimental group while there isn't improvement in control group .According to pain is higher improvement rate to patients live in rural and urban area in the experimental group than in control group.

Dailiana et al., (2015) reported that there aren't significant differences were present between rural and urban residences, and post-operative pain, depression and anxiety.

This can be explained that psycho-education was effective that led to higher improvement of anxiety, depression and pain in the experimental group than in control group.

The present result showed that there is highly significant association between marital status, preoperative and post-operative anxiety with highly improvement rate to single in the experimental group and married patients in control group while with depression there was higher improvement rate in single patients in the experimental than in control group. Also with pain there is higher improvement rate in married patients in the experimental group and divorced patients in control group.

These results are congruent with the study conducted by Dailiana et al., (2015) who found that single patients did not have worse post-operative anxiety, depression compared to married patients. 


\section{Conclusions}

Based on the results of the present study, it can be concluded that:

- All Participants (of both groups) had high level of depression, anxiety and pain intensity preoperative surgery.

- Preoperative psycho-education was highly effective in the experimental group with respect to postoperative depression, anxiety and pain.

- There are highly significant association between socio demographic characteristics, depression, anxiety and pain.

\section{Recommendations}

Based on findings of the present study, the following recommendations are suggested:

1- Liaison psychiatric nurse must be available to deal with psychological distress and impaired mental adjustment of patient's pre and postoperative surgery. They can provide help for patients to cope with depression and anxiety through training and relaxation techniques.

2- Psycho-educational interventions must be implemented for patients which include information about disease (causes and symptoms of disease), surgical process, surgical complications and how to deal with them .A booklet containing these information is recommended to be given to these patients as a reference during hospital stay and after discharge.

\section{Reference}

1. Blom, A., Artz, N., Beswick, A., Burston, A., Dieppe, P., Elvers, K., \& Lenguerrand, E., (2016): Measuring functional outcomes in patients having hip and knee replacement.

2. Boon, H., Gaboury, I., Balneaves, L., Tsui, T., Ng, J., \& Bozinovski, N., (2016): INCAM Research Symposium: Expanding PersonCentred Care through Integrative Health Research. Journal of Complementary and Integrative Medicine, Vol (13), No (4), PP. 27.

3. Burton, D., King, A., Bartley, J., Petrie, K., \& Broadbent, E., (2018): The surgical anxiety questionnaire (SAQ): development and validation. Psychology \& health, 1-18.

4. Chen,S. R., Chen, C., \& Lin, P., (2014): The effect of educational intervention on the pain and rehabilitation performance of patients who undergo a total knee replacement. Journal of clinical nursing, Vol(23), No(1-2), Pp.279-287.

5. Choi, Y., \& Ra, H., (2016): Patient satisfaction after total knee arthroplasty. Knee surgery \& related research, 28(1), 1.
6. Dailiana, Z., Papakostidou, I., Varitimidis, S., Liaropoulos, L., Zintzaras, E., Karachalios, T., \& Malizos, N., (2015): Patient-reported quality of life after primary major joint arthroplasty: a prospective comparison of hip and knee arthroplasty. BMC musculoskeletal disorders, Vol (16), NO (1), Pp.366.

7. Hamilton, D., Howie, C., Burnett, R., Simpson, A., \& Patton, J., (2015): Dealing with the predicted increase in demand for revision total knee arthroplasty: challenges, risks and opportunities. The bone \& joint journal, Vol(97),NO(6), Pp.723-728.

8. Hanusch B., O'Connor D., Ions P., Scott A., Gregg P., (2014): Effects of psychological distress and perceptions of illness on recovery from total knee replacement. Bone and Joint Journal. ; $\operatorname{Vol}(96), \operatorname{No}(2): 210$

216rthroplasty. The Knee, 23(6), 1044-1048.

9. Hoefnagels, M., (2014): Biology: concepts and investigations. McGraw-Hill Higher Education.

10. Kisner, C., Colby, L., \& Borstad, J., (2017): Therapeutic exercise: foundations and techniques. Fa Davis.

11. Krupic, F., Rolfson, O., Nemes, S., \& Kärrholm, J., (2016): Poor patient-reported outcome after hip replacement, related to poor perception of perioperative information, commoner in immigrants than in nonimmigrants. Acta orthopaedica, Vol (87), $\mathrm{NO}(3), P p$. 218-224.

12. Lindberg, M., Miaskowski, C., Rustøen, T., Rosseland, L., Paul, S., \& Lerdal, A., (2016): Preoperative pain, symptoms, and psychological factors related to higher acute pain trajectories during hospitalization for total knee arthroplasty. PloS one, $\operatorname{Vol}(11), N o(9), 0161681$.

13. Mottola C., (1993): Measurmentstrategies:the visual analogue scale Decubitus.; Vol(6),No(5):Pp.56-58.

14. Nawana, N., Gorhan, M., Frasier, W., Cranson, C., Serhan, H., O'neil, M., \& Selover, S., (2015): U.S. Patent Application No. 14/734,Pp.359.

15. Pasyar, N., Rambod, M., \& Kahkhaee, F., (2018): The Effect of Foot Massage on Pain Intensity and Anxiety in Patients Having Undergone a Tibial Shaft Fracture Surgery: A Randomized Clinical Trial. Journal of orthopaedic trauma, 32(12), e482-e486.

16. Riddle, D., Perera, R., Nay, W., \& Dumenci, L., (2015): What is the relationship between depressive symptoms and pain during functional tasks in persons undergoing TKA? A 6-year perioperative cohort study. Clinical 
Orthopaedics and Related Research ${ }$, Vol(473),No(11), Pp.3527-3534.

17. Rothrock, J., (2014): Alexander's Care of the Patient in Surgery-E-Book. Elsevier Health Sciences.

18. Scott, J., Mathias, J., \& Kneebone, A., (2016): Depression and anxiety after total joint replacement among older adults: a metaanalysis. Aging \& mental health, Vol(20),No(12), Pp.1243-1254.

19. Simeone, S., Pucciarelli, G., Perrone, M., Rea, T., Gargiulo, G., Dell'Angelo, G., \& Vosa, C., (2017): Comparative analysis: implementation of a pre-operative educational intervention to decrease anxiety among parents of children with congenital heart disease. Journal of pediatric nursing, 35, Pp.144-148.

20. Stoicea, N., Magal, S., Kim, J., Bai, M., Rogers, B., \& Bergese, S., (2018): Post-acute Transitional Journey: Caring for Orthopedic Surgery Patients. Frontiers in medicine, 5, 342.

21. Tang, X., Zhu, J., Zhou, Z., Shen, B., Kang, P., Pei, F., \& Li, J., (2016): Total hip replacement in adult patients with severe Kashin-Beck disease of the hip. BMC musculoskeletal disorders, Vol (17), NO (1), Pp.289.

22. Tristaino, V., Lantieri, F., Tornago, S., Gramazio, M., Carriere, E., \& Camera, A., (2016): Effectiveness of psychological support in patients undergoing primary total hip or knee arthroplasty: a controlled cohort study. Journal of Orthopaedics and Traumatology, Vol(17),No(2), Pp.137-147.

23. Vina, E., Ran, D., Ashbeck, E., Kaur, M., \& Kwoh, C., (2017): Relationship between knee pain and patient preferences for joint replacement: health care access matters. Arthritis care \& research, $\operatorname{Vol}(69), \operatorname{No}(1), 95-$ 103.

24. Waimann, C., Fernandez-Mazarambroz, R., Cantor, S., Lopez-Olivo, M., Barbo, A., Landon, G., \& Suarez-Almazor, M., (2016): Effect of body mass index and psychosocial traits on Total knee replacement costs in patients with osteoarthritis. The Journal of rheumatology, jrheum-151301

25. Wilson, C., Mitchelson, A., Tzeng, T., ElOthmani, M., Saleh, J., Vasdev, S., \& Saleh, K., (2016): Caring for the surgically anxious patient: a review of the interventions and a guide to optimizing surgical outcomes. The American Journal of Surgery, Vol(212), No(1), Pp.151-159.
26. Zigmond A., Snaith R., (1983): The Hospital Anxiety and Depression Scale.Acta Psychiatr Scand;67 :Pp.361-37. 